\title{
A Minimal Presentation of a Two-Generator Permutation Group on the Set of Integers
}

\author{
Simon Aloff ${ }^{*}{ }^{*}$, Michael Miniere ${ }^{1}$, John T. Saccoman ${ }^{2}$ \\ ${ }^{1}$ Middlesex College, Edison, NJ, USA \\ ${ }^{2}$ Seton Hall University, South Orange, NJ, USA \\ Email: *saloff@middlesexcc.edu
}

How to cite this paper: Aloff, S., Miniere, M. and Saccoman, J.T. (2021) A Minimal Presentation of a Two-Generator Permutation Group on the Set of Integers. Advances in Pure Mathematics, 11, 816-834. https://doi.org/10.4236/apm.2021.1110055

Received: September 20, 2021

Accepted: October 26, 2021

Published: October 29, 2021

Copyright (c) 2021 by author(s) and Scientific Research Publishing Inc. This work is licensed under the Creative Commons Attribution International License (CC BY 4.0).

http://creativecommons.org/licenses/by/4.0/

(c) (i) Open Access

\begin{abstract}
In this paper, we investigate the algebraic structure of certain 2-generator groups of permutations of the integers. The groups fall into two infinite classes: one class terminates with the quaternion group and the other class terminates with the Klein-four group. We show that all the groups are finitely presented and we determine minimal presentations in each case. Finally, we determine the order of each group.
\end{abstract}

\section{Keywords}

Permutation Groups, Combinatorial Group Theory, Presentation of Groups

\section{Introduction}

We determine finite minimal presentations for certain 2-generator groups of permutations of the integers. Much of the work contained herein appeared in [1]. For further results in this area, we recommend [2] and [3]. For all algebra definitions and terminology not found in this paper, we refer the reader to [4], and for more background on permutation groups, we refer the reader to [5].

First, we introduce some notation that we will follow in this paper. We will denote by $\mathbb{Z}^{+}$and $\mathbb{Z}^{-}$two separate copies of the integers, i.e., $\mathbb{Z}^{+}=\left\{\cdots,-3^{+},-2^{+},-1^{+}, 0^{+}, 1^{+}, 2^{+}, 3^{+}, \cdots\right\}, \mathbb{Z}^{-}=\left\{\cdots,-3^{-},-2^{-},-1^{-}, 0^{-}, 1^{-}, 2^{-}, 3^{-}, \cdots\right\}$, and let $S=\mathbb{Z}^{+} \cup \mathbb{Z}^{-}$. We denote by $\Sigma$ the group of all one-to-one mappings of $S$ onto itself. We will refer to $\Sigma$ as the infinite symmetric group, and its elements will be called permutations of $S$. This paper will, for the most part, deal with the combinatorial group theory aspects of the permutation group $G$ generated by $\sigma$ and $\tau$. In our notation, $\sigma \tau$ denotes $\tau$ followed by $\sigma$. The permutations $\sigma, \tau \in \Sigma$ that are the focus of this work are defined as follows: 
- $\sigma\left(x^{+}\right)=(x-1)^{+}$

- $\sigma\left(x^{-}\right)=(x+1)^{-}$

- $\tau\left(x^{-}\right)=x^{+}$

- $\tau\left(x^{+}\right)= \begin{cases}(x+2)^{-} & x \equiv 0(\bmod 2) \\ (x-2)^{-} & x \equiv 1(\bmod 2)\end{cases}$

We illustrate $\sigma$ and $\tau$ in Figure 1 .

We state a few pertinent definitions.

Definition 1.1 Let $\tilde{G}$ be an arbitrary group.

1) A group $\tilde{G}$ is finitely generated by elements $g_{1}, g_{2}, \cdots, g_{j} \in \tilde{G}$ if each $x \in \tilde{G}$ has a representation $x=x_{1} x_{2} \cdots x_{n}$ with each $x_{i} \in\left\{g_{1}, g_{1}^{-1}, g_{2}, g_{2}^{-1}, \cdots, g_{j}, g_{j}^{-1}\right\}$. For the following definitions, we assume that $\tilde{G}$ has a specified set of generators $g_{1}, g_{2}, \cdots, g_{j}$.

2) A word in $\tilde{G}$ is a sequence $x_{1}, x_{2}, \cdots, x_{n}$ with each $x_{i} \in\left\{g_{1}, g_{1}^{-1}, g_{2}, g_{2}^{-1}, \cdots, g_{j}, g_{j}^{-1}\right\}$. The word $w=x_{1}, x_{2}, \cdots, x_{n}$ represents the element $x_{1} x_{2} \cdots x_{n} \in \tilde{G}$, so we will write $w=x_{1} x_{2} \cdots x_{n}$. We will allow the empty word (no symbols) which represents the identity in $\tilde{G}$.

3) If $w=x_{1} x_{2} \cdots x_{n}$ is a word, then $w^{-1}=x_{n}^{-1} x_{n-1}^{-1} \cdots x_{1}^{-1}$.

4) A relator is a word that represents the identity. A trivial relator is a word $w=x x^{-1}$ with $x$ a word. The set of relators is denoted $R(\tilde{G})$.

5) Two words are equivalent if one can be transformed into the other in a finite number of steps by inserting or deleting a trivial relator at an arbitrary location during each step. This is a valid equivalence relation on the set of words. Hereafter, a word will mean the equivalence class of the word.

6) If $x, y$ are words, then $x^{-1} y x$ is a conjugate of $y$. The conjugate of a relator is itself a relator, and a finite product of relators is also a relator.

7) A relator $z$ can be inserted into a word $\omega_{1}=A B$ (with $A, B$ words) to obtain a new word $\omega_{2}=A z B$ by multiplying $\omega_{1}$ on the right by $B^{-1} z B$, i.e., $\omega_{1}=A B \rightarrow A B\left(B^{-1} z B\right)=A z B=\omega_{2}$.

8) If $\omega_{1}, \omega_{2}, \cdots, \omega_{n}$ are words, then $N\left(\omega_{1}, \omega_{2}, \cdots, \omega_{n}\right)$ is the set of words that are the finite products of the conjugates of $\omega_{1}, \omega_{1}^{-1}, \omega_{2}, \omega_{2}^{-1}, \cdots, \omega_{n}, \omega_{n}^{-1}$. If each $\omega_{i} \in R(\tilde{G})$, then $N\left(\omega_{1}, \omega_{2}, \cdots, \omega_{n}\right) \subset R(\tilde{G})$.

9) $\tilde{G}$ is finitely presented if there is a finite set of relators $\left\{z_{1}, z_{2}, \cdots, z_{n}\right\}$ such that $R(\tilde{G})=N\left(z_{1}, z_{2}, \cdots, z_{n}\right)$. The presentation $\left\{z_{1}, z_{2}, \cdots, z_{n}\right\}$ is minimal if $R(\tilde{G}) \neq N\left(z_{1}, z_{2}, \cdots, z_{i-1}, z_{i+1}, \cdots, z_{n}\right)$ for $i=1,2, \cdots, n$.

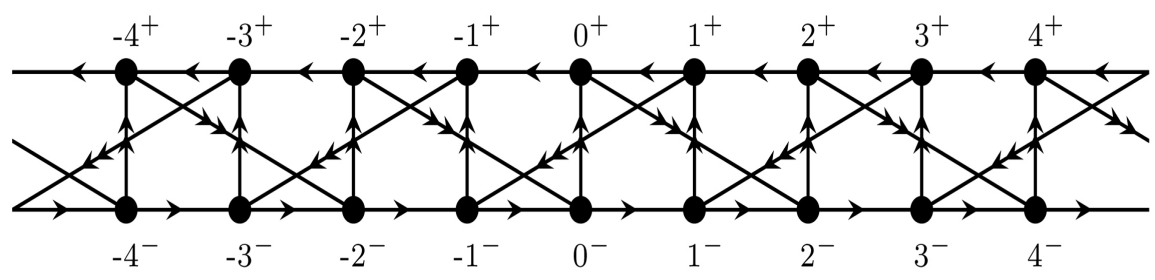

Figure 1. The permutation $\sigma$ is represented by the single arrow, and $\tau$ is represented by the double arrow. 


\section{Main Results}

\subsection{Properties of $\tau$ and $\sigma$}

Theorems 2.1 and 2.2 demonstrate how the application of $\tau$ and $\sigma$ to integers impacts the resulting parities.

Theorem 2.1 The permutations $\tau$ and $\sigma$ satisfy the following properties:

1) If $x \equiv 0(\bmod 2)$, then

- $\tau^{2}\left(x^{+}\right)=(x+2)^{+}$, and $\tau^{-2}\left(x^{+}\right)=(x-2)^{+}$

- $\tau^{2}\left(x^{-}\right)=(x+2)^{-}$, and $\tau^{-2}\left(x^{-}\right)=(x-2)^{-}$

- $(\tau \sigma)^{2}\left(x^{+}\right)=(x-2)^{+}$, and $(\tau \sigma)^{-2}\left(x^{+}\right)=(x+2)^{+}$

- $(\tau \sigma)^{2}\left(x^{-}\right)=(x+2)^{-}$, and $(\tau \sigma)^{-2}\left(x^{-}\right)=(x-2)^{-}$

2) If $x \equiv 1(\bmod 2)$, then

$\tau^{2}\left(x^{+}\right)=(x-2)^{+}$, and $\tau^{-2}\left(x^{+}\right)=(x+2)^{+}$

- $\tau^{2}\left(x^{-}\right)=(x-2)^{-}$, and $\tau^{-2}\left(x^{-}\right)=(x+2)^{-}$

- $(\tau \sigma)^{2}\left(x^{+}\right)=(x+2)^{+}$, and $(\tau \sigma)^{-2}\left(x^{-}\right)=(x-2)^{+}$

- $(\tau \sigma)^{2}\left(x^{-}\right)=(x-2)^{-}$, and $(\tau \sigma)^{-2}\left(x^{+}\right)=(x+2)^{-}$.

Proof: (1.) Suppose $x \equiv 0(\bmod 2)$. We prove the first formula:

- $\tau^{2}\left(x^{+}\right)=\tau\left((x+2)^{-}\right)=(x+2)^{+}$,

- $\tau^{2}\left(x^{-}\right)=\tau\left(x^{+}\right)=(x+2)^{-}$,

- $\tau \sigma \tau \sigma\left(x^{+}\right)=\tau \sigma \tau\left((x-1)^{+}\right)=\tau \sigma\left((x-3)^{-}\right)=\tau\left((x-2)^{-}\right)=(x-2)^{+}$,

- $\tau \sigma \tau \sigma\left(x^{-}\right)=\tau \sigma \tau\left((x+1)^{-}\right)=\tau \sigma\left((x+1)^{+}\right)=\tau\left(x^{+}\right)=(x+2)^{-}$.

To determine inverses, note that $\tau^{2}$ and $(\tau \sigma)^{2}$ preserve both sign parity (i.e.,,+- ) and even/odd parity. If $\tau^{-2}\left(x^{+}\right)=y^{+}$, then $x^{+}=\tau^{2}\left(y^{+}\right)=(y+2)^{+}$, so $y=x-2$, and thus, $\tau^{-2}\left(x^{+}\right)=(x-2)^{+}$. The proofs of the other formulas are similar. (2.) Suppose $x \equiv 1(\bmod 2)$. We prove the first formula:

- $\tau^{2}\left(x^{+}\right)=\tau\left((x-2)^{-}\right)=(x-2)^{+}$,

- $\tau^{2}\left(x^{-}\right)=\tau\left(x^{+}\right)=(x-2)^{-}$,

- $\tau \sigma \tau \sigma\left(x^{+}\right)=\tau \sigma \tau\left((x-1)^{+}\right)=\tau \sigma\left((x+1)^{-}\right)=\tau\left((x+2)^{-}\right)=(x+2)^{+}$,

- $\tau \sigma \tau \sigma\left(x^{-}\right)=\tau \sigma \tau\left((x+1)^{-}\right)=\tau \sigma\left((x+1)^{+}\right)=\tau\left(x^{+}\right)=(x-2)^{-}$.

The inverse properties follow as in part (1.), and the other formulas follow similarly.

The next theorem generalizes Theorem 2.1 .

Theorem 2.2 Let $a, b, c \in \mathbb{Z}$ and $x \in 2 \mathbb{Z}$.

$1)$.

- $(\tau \sigma)^{a}\left(x^{+}\right)=\left\{\begin{array}{cc}(x-a)^{+}, & a \equiv 0(\bmod 2) \\ (x-a-2)^{-}, & a \equiv 1(\bmod 2)\end{array}\right.$

- $(\tau \sigma)^{a}\left((x+1)^{+}\right)= \begin{cases}(x+1+a)^{+}, & a \equiv 0(\bmod 2) \\ (x+1+a)^{-}, & a \equiv 1(\bmod 2)\end{cases}$

- $(\tau \sigma)^{a}\left(x^{-}\right)= \begin{cases}(x+a)^{-}, & a \equiv 0(\bmod 2) \\ (x+a)^{+}, & a \equiv 1(\bmod 2)\end{cases}$ 
- $(\tau \sigma)^{a}\left((x+1)^{-}\right)= \begin{cases}(x+1-a)^{-}, & a \equiv 0(\bmod 2) \\ (x+3-a)^{+}, & a \equiv 1(\bmod 2)\end{cases}$

2).

- $\sigma^{b} \tau^{c}\left(x^{+}\right)=\left\{\begin{array}{cc}(x-b+c)^{+}, & c \equiv 0(\bmod 2) \\ (x+1+b+c)^{-}, & c \equiv 1(\bmod 2)\end{array}\right.$

- $\sigma^{b} \tau^{c}\left((x+1)^{+}\right)=\left\{\begin{array}{cc}(x+1-b-c)^{+}, & c \equiv 0(\bmod 2) \\ (x+b-c)^{-}, & c \equiv 1(\bmod 2)\end{array}\right.$

- $\sigma^{b} \tau^{c}\left(x^{-}\right)=\left\{\begin{array}{cc}(x+b+c)^{-}, & c \equiv 0(\bmod 2) \\ (x-b+c-1)^{+}, & c \equiv 1(\bmod 2)\end{array}\right.$

- $\sigma^{b} \tau^{c}\left((x+1)^{-}\right)= \begin{cases}(x+1+b-c)^{-}, & c \equiv 0(\bmod 2) \\ (x-b-c+2)^{+}, & c \equiv 1(\bmod 2)\end{cases}$

Proof:

1) First, suppose $a \equiv 0(\bmod 2)$, so $a=2 k$. By Theorem 2.1,

- $(\tau \sigma)^{a}\left(x^{+}\right)=\left[(\tau \sigma)^{2}\right]^{k}\left(x^{+}\right)=(x-2 k)^{+}=(x-a)^{+}$

- $(\tau \sigma)^{a}\left(x^{-}\right)=\left[(\tau \sigma)^{2}\right]^{k}\left(x^{-}\right)=(x+2 k)^{-}=(x+a)^{-}$

- $(\tau \sigma)^{a}\left((x+1)^{+}\right)=\left[(\tau \sigma)^{2}\right]^{k}\left((x+1)^{+}\right)=(x+1+2 k)^{+}=(x+1+a)^{+}$

- $(\tau \sigma)^{a}\left((x+1)^{-}\right)=\left[(\tau \sigma)^{2}\right]^{k}\left((x+1)^{-}\right)=(x+1-2 k)^{-}=(x+1-a)^{-}$

Now suppose $a \equiv 1(\bmod 2)$, so $a=2 k+1$. Then

- $(\tau \sigma)^{a}\left(x^{+}\right)=(\tau \sigma)(\tau \sigma)^{2 k}\left(x^{+}\right)=(\tau \sigma)\left((x-2 k)^{+}\right)=(x-2 k-3)^{-}=(x-a-2)^{-}$

- $(\tau \sigma)^{a}\left(x^{-}\right)=(\tau \sigma)(\tau \sigma)^{2 k}\left(x^{-}\right)=(\tau \sigma)\left((x+2 k)^{-}\right)=(x+2 k+1)^{+}=(x+a)^{+}$

- $(\tau \sigma)^{a}\left((x+1)^{+}\right)=(\tau \sigma)(\tau \sigma)^{2 k}\left((x+1)^{+}\right)=(\tau \sigma)\left((x+1+2 k)^{+}\right)$

$$
=(x+2 k+2)^{-}=(x+1+a)^{-}
$$

$(\tau \sigma)^{a}\left((x+1)^{-}\right)=(\tau \sigma)(\tau \sigma)^{2 k}\left((x+1)^{-}\right)=(\tau \sigma)\left((x+1-2 k)^{-}\right)$

$$
=(x+2-2 k)^{+}=(x+3-a)^{+}
$$

2) Suppose $c \equiv 0(\bmod 2)$, so $c=2 k$. Then

- $\sigma^{b} \tau^{c}\left(x^{+}\right)=\sigma^{b}\left(\tau^{2}\right)^{k}\left(x^{+}\right)=\sigma^{b}\left((x+2 k)^{+}\right)=(x+2 k-b)^{+}=(x-b+c)^{+}$

- $\sigma^{b} \tau^{c}\left(x^{-}\right)=\sigma^{b}\left(\tau^{2}\right)^{k}\left(x^{-}\right)=\sigma^{b}\left((x+2 k)^{-}\right)=(x+2 k+b)^{-}=(x+b+c)^{-}$

- $\sigma^{b} \tau^{c}\left((x+1)^{+}\right)=\sigma^{b}\left(\tau^{2}\right)^{k}\left((x+1)^{+}\right)=\sigma^{b}\left((x+1-2 k)^{+}\right)$

$$
=(x+1-b-2 k)^{+}=(x+1-b-c)^{+}
$$

- $\sigma^{b} \tau^{c}\left((x+1)^{-}\right)=\sigma^{b}\left(\tau^{2}\right)^{k}\left((x+1)^{-}\right)=\sigma^{b}\left((x+1-2 k)^{-}\right)$.

$$
=(x+1+b-2 k)^{-}=(x+1+b-c)^{-}
$$

Also, if $c \equiv 1(\bmod 2)$, so $c=2 k+1$, then 


$$
\begin{aligned}
\sigma^{b} \tau^{c}\left(x^{+}\right) & =\sigma^{b} \tau \tau^{2 k}\left(x^{+}\right)=\sigma^{b} \tau\left((x+2 k)^{+}\right)=\sigma^{b}\left((x+2 k+2)^{-}\right) \\
= & (x+2 k+2+b)^{-}=(x+b+c+1)^{-} \\
\sigma^{b} \tau^{c}\left(x^{-}\right) & =\sigma^{b} \tau \tau^{2 k}\left(x^{-}\right)=\sigma^{b} \tau\left((x+2 k)^{-}\right)=\sigma^{b}\left((x+2 k)^{+}\right) \\
=(x+2 k-b)^{+}=(x-b+c-1)^{+} & \\
\sigma^{b} \tau^{c}\left((x+1)^{+}\right) & =\sigma^{b} \tau \tau^{2 k}\left((x+1)^{+}\right)=\sigma^{b} \tau\left((x+1-2 k)^{+}\right) \\
& =\sigma^{b}\left((x-1-2 k)^{-}\right)=(x+b-1-2 k)^{-}=(x+b-c)^{-} \\
\sigma^{b} \tau^{c}\left((x+1)^{-}\right) & =\sigma^{b} \tau \tau^{2 k}\left((x+1)^{-}\right)=\sigma^{b} \tau\left((x+1-2 k)^{-}\right) \\
& =\sigma^{b}\left((x+1-2 k)^{+}\right)=(x+1-2 k-b)^{+}=(x-b-c+2)^{+}
\end{aligned}
$$

We can use Theorem 2.2 to prove a uniqueness of representation theorem for the permutations $\sigma, \tau$.

Theorem 2.3 Let $a, b, c \in \mathbb{Z}$ and $a \geq 0$. Suppose $(\tau \sigma)^{a}=\sigma^{b} \tau^{c}$. Then $a=b=c=0$.

Proof. If $(\tau \sigma)^{a}=\sigma^{b} \tau^{c}$, then their images agree on all values in $S$. In particular, on $0^{+}, 1^{+}, 0^{-}$and $1^{-}$. There are two cases:

1) First suppose $a \equiv 0(\bmod 2)$. Then we must have $c \equiv 0(\bmod 2)$ in order to preserve sign parity. We substitute the values $0^{+}, 1^{+}, 0^{-}$and $1^{-}$into the equation $(\tau \sigma)^{a}=\sigma^{b} \tau^{c}$ and use Theorem 2.2. This yields

- At $0^{+}$, have $-a=-b+c$.

- At $1^{+}$, have $a+1=-b-c+1$.

- At $0^{-}$, have $a=b+c$.

- At $1^{-}$, have $-a+1=b-c+1$.

These equations imply that $a=b=c=0$.

2) Now suppose that $a \equiv 1(\bmod 2)$, and again substitute the values $0^{+}, 1^{+}, 0^{-}$ and $1^{-}$, respectively. Now,

- At $0^{+}$, have $-a-2=b+c+1$.

- At $1^{+}$, have $a+1=b-c$.

- At $0^{-}$, have $a=-b+c-1$.

- At $1^{-}$, have $-a+3=-b-c+2$.

These equations imply that $a=b=c=-1$. Since we assumed $a \geq 0$, this is impossible, so the desired result follows.

Now we return to the group $G$ generated by the permutations $\sigma, \tau, \sigma^{-1}$ and $\tau^{-1}$. We will show that $G$ is finitely presented and determine a minimal presentation.

Theorem 2.4 The following words are in $R(G)$.

- $\omega_{1}=\tau^{-1} \sigma^{2} \tau \sigma^{2}$

- $\omega_{2}=\sigma^{-1} \tau^{2} \sigma \tau^{2}$

- $\omega_{3}=\sigma^{2} \omega_{1}^{-1} \sigma^{-2}=\tau^{-1} \sigma^{-2} \tau \sigma^{-2}$

- $\omega_{4}=\sigma^{-2} \tau \omega_{1} \tau^{-1} \sigma^{2}=\tau \sigma^{2} \tau^{-1} \sigma^{2}$

- $\omega_{5}=\tau \omega_{1}^{-1} \tau^{-1}=\tau \sigma^{-2} \tau^{-1} \sigma^{-2}$

- $\omega_{6}=\sigma \omega_{2} \sigma^{-1}=\tau^{2} \sigma \tau^{2} \sigma^{-1}$ 
- $\omega_{7}=\tau^{2} \omega_{2} \tau^{-2}=\tau^{2} \sigma^{-1} \tau^{2} \sigma$

- $\omega_{8}=\tau^{-2} \sigma \omega_{2}^{-1} \sigma^{-1} \tau^{2}=\tau^{-2} \sigma \tau^{-2} \sigma^{-1}$

Then $N\left(\omega_{1}, \omega_{2}\right)=R(G)$, and $\left\{\omega_{1}, \omega_{2}\right\}$ is a finite presentation for $G$.

Proof. We will prove this theorem by demonstrating containment in both directions. To show $N\left(\omega_{1}, \omega_{2}\right) \subseteq R(G)$, we first show that $\omega_{1}$ and $\omega_{2}$ are in $R(G)$. If $x \equiv 0(\bmod 2)$, then

- $\omega_{1}\left(x^{+}\right)=\tau^{-1} \sigma^{2} \tau \sigma^{2}\left(x^{+}\right)=\tau^{-1} \sigma^{2} \tau\left((x-2)^{+}\right)=\tau^{-1} \sigma^{2}\left(x^{-}\right)=\tau^{-1}\left((x+2)^{-}\right)=x^{+}$

$$
\begin{aligned}
& \begin{aligned}
\omega_{2}\left(x^{+}\right) & =\sigma^{-1} \tau^{2} \sigma \tau^{2}\left(x^{+}\right)=\sigma^{-1} \tau^{2} \sigma\left((x+2)^{+}\right)=\sigma^{-1} \tau^{2}\left((x+1)^{+}\right) \\
& =\sigma^{-1}\left((x-1)^{+}\right)=x^{+}
\end{aligned} \\
& \begin{aligned}
\omega_{1}\left(x^{-}\right) & =\tau^{-1} \sigma^{2} \tau \sigma^{2}\left(x^{-}\right)=\tau^{-1} \sigma^{2} \tau\left((x+2)^{-}\right)=\tau^{-1} \sigma^{2}\left((x+2)^{+}\right) \\
& =\tau^{-1}\left(x^{+}\right)=x^{-}
\end{aligned} \\
& \begin{aligned}
\omega_{2}\left(x^{-}\right) & =\sigma^{-1} \tau^{2} \sigma \tau^{2}\left(x^{-}\right)=\sigma^{-1} \tau^{2} \sigma\left((x+2)^{-}\right)=\sigma^{-1} \tau^{2}\left((x+3)^{-}\right) \\
& =\sigma^{-1}\left((x+1)^{-}\right)=x^{-}
\end{aligned} \\
& \begin{aligned}
\omega_{1}\left(x^{+}\right) & =\tau^{-1} \sigma^{2} \tau \sigma^{2}\left(x^{+}\right)=\tau^{-1} \sigma^{2} \tau\left((x-2)^{+}\right)=\tau^{-1} \sigma^{2}\left((x-4)^{-}\right) \\
& =\tau^{-1}\left((x-2)^{-}\right)=x^{+}
\end{aligned} \\
& \begin{aligned}
\omega_{2}\left(x^{+}\right) & =\sigma^{-1} \tau^{2} \sigma \tau^{2}\left(x^{+}\right)=\sigma^{-1} \tau^{2} \sigma\left((x-2)^{+}\right)=\sigma^{-1} \tau^{2}\left((x-3)^{+}\right) \\
& =\sigma^{-1}\left((x-1)^{+}\right)=x^{+}
\end{aligned} \\
& \begin{aligned}
\omega_{1}\left(x^{-}\right) & =\tau^{-1} \sigma^{2} \tau \sigma^{2}\left(x^{-}\right)=\tau^{-1} \sigma^{2} \tau\left((x+2)^{-}\right)=\tau^{-1} \sigma^{2}\left((x+2)^{+}\right) \\
& =\tau^{-1}\left(x^{+}\right)=x^{-}
\end{aligned} \\
& \omega_{2}\left(x^{-}\right)=\sigma^{-1} \tau^{2} \sigma \tau^{2}\left(x^{-}\right)=\sigma^{-1} \tau^{2} \sigma\left((x-2)^{-}\right)=\sigma^{-1} \tau^{2}\left((x-1)^{-}\right) \\
& =\sigma^{-1}\left((x+1)^{-}\right)=x^{-}
\end{aligned}
$$

Since the conjugate of an element in $R(G)$ is also in $R(G)$, it follows that $N\left(\omega_{1}, \omega_{2}\right) \subseteq R(G)$. In addition, since $\omega_{3}, \omega_{4}, \cdots, \omega_{8}$ are conjugates of $\omega_{1}, \omega_{1}^{-1}, \omega_{2}$, or $\omega_{2}^{-1}$, they are in $R(G)$.

Now, to show that the reverse inclusion holds, we assume that $g$ is a word in $G$ having the form $g=A x y B$, where $A, B$ are words in $G$. Then $g$ can be transformed to $\tilde{g}=A \tilde{y} \tilde{x} B$ by multiplying $g$ on the right by $B^{-1} y^{-1} z y B$ where $x, \tilde{x}, y, \tilde{y}, z$ are as indicated below:

\begin{tabular}{ccccc}
\hline$x$ & $y$ & $\tilde{y}$ & $\tilde{x}$ & $z$ \\
\hline$\tau$ & $\sigma^{-2}$ & $\sigma^{2}$ & $\tau$ & $\omega_{1}$ \\
$\sigma^{2}$ & $\tau^{-1}$ & $\tau^{-1}$ & $\sigma^{-2}$ & $\omega_{1}^{-1}$ \\
$\sigma$ & $\tau^{-2}$ & $\tau^{2}$ & $\sigma$ & $\omega_{2}$ \\
$\tau^{2}$ & $\sigma^{-1}$ & $\sigma^{-1}$ & $\tau^{-2}$ & $\omega_{2}^{-1}$ \\
\hline
\end{tabular}




\section{Continued}

\begin{tabular}{ccccc}
\hline$\tau$ & $\sigma^{2}$ & $\sigma^{-2}$ & $\tau$ & $\omega_{3}$ \\
$\sigma^{-2}$ & $\tau^{-1}$ & $\tau^{-1}$ & $\sigma^{2}$ & $\omega_{3}^{-1}$ \\
$\tau^{-1}$ & $\sigma^{-2}$ & $\sigma^{2}$ & $\tau^{-1}$ & $\omega_{4}$ \\
$\sigma^{2}$ & $\tau$ & $\tau$ & $\sigma^{-2}$ & $\omega_{4}^{-1}$ \\
\hline$\tau^{-1}$ & $\sigma^{2}$ & $\sigma^{-2}$ & $\tau^{-1}$ & $\omega_{5}$ \\
$\sigma^{-2}$ & $\tau$ & $\tau$ & $\sigma^{2}$ & $\omega_{5}^{-1}$ \\
$\tau^{-2}$ & $\sigma$ & $\sigma$ & $\tau^{2}$ & $\omega_{6}^{-1}$ \\
$\sigma^{-1}$ & $\tau^{2}$ & $\tau^{-2}$ & $\sigma^{-1}$ & $\omega_{7}$ \\
$\tau^{-2}$ & $\sigma^{-1}$ & $\tau^{-2}$ & $\sigma^{-1}$ & $\omega_{7}^{-1}$ \\
$\sigma$ & $\tau^{2}$ & $\tau^{-2}$ & $\sigma$ & $\omega_{8}$ \\
$\tau^{2}$ & $\sigma$ & $\sigma$ & $\tau^{-2}$ & $\omega_{8}^{-1}$ \\
\hline$\sigma^{-1}$ & $\tau^{-2}$ & $\tau^{2}$ & $\sigma^{-1}$ & \\
\hline
\end{tabular}

Hence, $\tilde{g}=g B^{-1} y^{-1} z y B$, implying that $g=\tilde{g} B^{-1} y^{-1} z^{-1} y B$. Since $B^{-1} y^{-1} z^{-1} y B \in N\left(\omega_{1}, \omega_{2}\right)$, this shows that any word $g \in G$ can be transformed to an element $\tilde{g}$ by moving even powers of $\sigma$ to the left and even powers of $\tau$ to the right. When this process is completed, $\tilde{g}$ has the form $\sigma^{b^{\prime}} x \tau^{c^{\prime}}$, where $x$ has the form $\tau^{i_{1}} \sigma^{j_{1}} \tau^{i_{2}} \sigma^{j_{2}} \cdots \tau^{i_{n}} \sigma^{j_{n}}$, with each $i_{k}, j_{k}= \pm 1$. If some exponent is -1 , say, $\tau^{-1}$, then we can represent this as $\tau^{-1}=\tau \tau^{-2}$. Now, the $\tau^{-2}$ can be moved to the right. In this way, we eventually arrive at $\tilde{g}=\sigma^{b}(\tau \sigma)^{a} \tau^{c}, a \geq 0$, and $\tilde{g}=g C$, with $C \in N\left(\omega_{1}, \omega_{2}\right)$. Now, suppose $g \in R(G)$. Since $C \in N\left(\omega_{1}, \omega_{2}\right)$, it follows that $C \in R(G)$. Therefore, $\tilde{g} \in R(G)$, so $\sigma^{b}(\tau \sigma)^{a} \tau^{c}$ is the identity, and $(\tau \sigma)^{a}=\sigma^{-b} \tau^{-c}$ in $G$. By Theorem 2.3, $a=b=c=0$. This means that $\tilde{g}$ is the empty word, and $g$ is the word $C^{-1} \in N\left(\omega_{1}, \omega_{2}\right)$. Thus, $R(G) \subseteq N\left(\omega_{1}, \omega_{2}\right)$, and we have $R(G)=N\left(\omega_{1}, \omega_{2}\right)$.

Theorem 2.4 has an immediate corollary.

Corollary 2.5 Every word $\omega \in G$ has an equivalent form $\omega=\sigma^{b}(\tau \sigma)^{a} \tau^{c} x$, where $x \in N\left(\omega_{1}, \omega_{2}\right)$. and $a \geq 0$.

We are now ready to prove our main result.

Theorem $2.6\left\{\omega_{1}, \omega_{2}\right\}$ is a minimal presentation for $G$.

Proof. We must show that $\omega_{1} \notin N\left(\omega_{2}\right)$ and $\omega_{2} \notin N\left(\omega_{1}\right)$, where $\omega_{1}=\tau^{-1} \sigma^{2} \tau \sigma^{2}$ and $\omega_{2}=\sigma^{-1} \tau^{2} \sigma \tau^{2}$. For any word $x=\sigma^{i_{1}} \tau^{j_{1}} \cdots \sigma^{i_{n}} \tau^{j_{n}}$, define $e_{\sigma}(x)=i_{1}+i_{2}+\cdots+i_{n}$, and $e_{\tau}(x)=j_{1}+j_{2}+\cdots+j_{n}$. If $y=z^{-1} \omega_{1} z$, then $e_{\sigma}(y)=4$. If $y=z^{-1} \omega_{2} z$, then $e_{\tau}(y)=4$. If $y=z^{-1} \omega_{1}^{-1} z$, then $e_{\sigma}(y)=-4$. If $y=z^{-1} \omega_{2}^{-1} z$, then $e_{\tau}(y)=-4$. If $x$ is a conjugate of $\omega_{2}$ or $\omega_{2}^{-1}$, then $e_{\sigma}(x)=0$. If $\omega_{1} \in N\left(\omega_{2}\right)$, then $\omega_{1}=x_{1} x_{2} \cdots x_{n}$, with each $x_{i}$ a conjugate of $\omega_{2}$ or $\omega_{2}^{-1}$. Therefore, $4=e_{\sigma}\left(\omega_{1}\right)=e_{\sigma}\left(x_{1}\right)+e_{\sigma}\left(x_{2}\right)+\cdots+e_{\sigma}\left(x_{n}\right)=0$, a contradiction. Hence, $\omega_{1} \notin N\left(\omega_{2}\right)$. By a similar argument, $\omega_{2} \notin N\left(\omega_{1}\right)$. 
Example 2.7 Transform $\omega=\tau^{4} \sigma^{-1} \tau \sigma^{2} \tau^{3} \sigma^{3}$ by $A x y B \rightarrow A \tilde{y} \tilde{x} B$ of Theorem 2.5 and show that $\omega \in N\left(\omega_{1}, \omega_{2}\right)$.

The step-by-step process involves multiplying $A x y B$ on the right by $C=B^{-1} y^{-1} z y B \quad$ (where $z$ is a particular value of $\omega_{i}$ or $\omega_{i}^{-1}$ ) to obtain Aỹ $\tilde{x} B$. At each stage, we make use of the reductions $\sigma^{a} \sigma^{b}=\sigma^{a+b}, \tau^{a} \tau^{b}=\tau^{a+b}$, delete $\sigma^{0}, \tau^{0}$. We halt the procedure when the identity, i.e., the empty word, is reached.

\begin{tabular}{cccccccc}
\hline$A$ & $x$ & $y$ & $B$ & $\tilde{y}$ & $\tilde{x}$ & $z$ & $C=B^{-1} y^{-1} z y B$ \\
\hline$A_{1}=\tau^{2}$ & $\tau^{2}$ & $\sigma^{-1}$ & $\tau \sigma^{2} \tau^{3} \sigma^{3}=B_{1}$ & $\sigma^{-1}$ & $\tau^{-2}$ & $\omega_{2}^{-1}$ & $C_{1}=B_{1}^{-1} \sigma \omega_{2}^{-1} \sigma^{-1} B_{1}$ \\
$A_{2}=$ empty & $\tau^{2}$ & $\sigma^{-1}$ & $\tau^{-1} \sigma^{2} \tau^{3} \sigma^{3}=B_{2}$ & $\sigma^{-1}$ & $\tau^{-2}$ & $\omega_{2}^{-1}$ & $C_{2}=B_{2}^{-1} \sigma \omega_{2}^{-1} \sigma^{-1} B_{2}$ \\
$A_{3}=\sigma^{-1} \tau^{-2}$ & $\tau^{-1}$ & $\sigma^{2}$ & $\tau^{3} \sigma^{3}=B_{3}$ & $\sigma^{-2}$ & $\tau^{-1}$ & $\omega_{5}$ & $C_{3}=B_{3}^{-1} \sigma^{-2} \omega_{5} \sigma^{2} B_{3}$ \\
$A_{4}=\sigma^{-1} \tau^{-1}$ & $\tau^{-1}$ & $\sigma^{-2}$ & $\tau^{2} \sigma^{3}=B_{4}$ & $\sigma^{2}$ & $\tau^{-1}$ & $\omega_{4}$ & $C_{4}=B_{4}^{-1} \sigma^{2} \omega_{4} \sigma^{-2} B_{4}$ \\
$A_{5}=\sigma^{-1}$ & $\tau^{-1}$ & $\sigma^{2}$ & $\tau \sigma^{3}=B_{5}$ & $\sigma^{-2}$ & $\tau^{-1}$ & $\omega_{5}$ & $C_{5}=B_{5}^{-1} \sigma^{-2} \omega_{5} \sigma^{2} B_{5}$ \\
\hline
\end{tabular}

So the first step replaces $\tau^{2} \sigma^{-1}$ with $\sigma^{-1} \tau^{-2}$ and simplifies by reducing exponents. At the final step, we obtain $\omega C_{1} C_{2} C_{3} C_{4} C_{5}=I$ (i.e., the empty word). Hence, $\omega=\left(C_{1} C_{2} C_{3} C_{4} C_{5}\right)^{-1}=C_{5}^{-1} C_{4}^{-1} C_{3}^{-1} C_{2}^{-1} C_{1}^{-1}$. Expressing everything in terms of powers of $\sigma$ and $\tau$ reduces the equation to $\omega=\tau^{4} \sigma^{-1} \tau \sigma^{2} \tau^{3} \sigma^{3}$, with deletion of $\sigma^{0}, \tau^{0}$.

\subsection{The Finite Permutation Groups $G_{4 \mathrm{n}}$}

We define a translation operation on $S=\mathbb{Z}^{+} \cup \mathbb{Z}^{-}$for each positive integer $n$. We then form equivalence classes denoted by $S_{4 n}$. The permutations $\sigma, \tau$ are well-defined mappings on $S_{4 n}$. The permutation group generated by $\sigma, \tau$ on $S_{4 n}$ is denoted by $G_{4 n}$. Theorem 2.2 is generalized in order to determine the relators $R\left(G_{4 n}\right)$ of $G_{4 n}$. We also determine the order of each $G_{4 n}$.

Definition 2.8 Let $n$ be a positive integer and $S=\mathbb{Z}^{+} \cup \mathbb{Z}^{-}$. Define a mapping $\phi_{n}: S \rightarrow S$ by

- $\phi_{n}\left(x^{+}\right)=(x+n)^{+}=\sigma^{-n}\left(x^{+}\right)$

- $\phi_{n}\left(x^{-}\right)=(x+n)^{-}=\sigma^{n}\left(x^{-}\right)$

If $x \in S$, then $x+n$ denotes $\phi_{n}(x)$.

Theorem 2.9 If $n$ is even, then $\sigma(x+n)=\sigma(x)+n$, and $\tau(x+n)=\tau(x)+n$ Proof. If $x=x^{+}$, then $\sigma\left(x^{+}+n\right)=\sigma\left((x+n)^{+}\right)=(x+n-1)^{+}=\sigma\left(x^{+}\right)+n$, and

$$
\tau\left(x^{+}+n\right)=\tau\left((x+n)^{+}\right)= \begin{cases}(x+n+2)^{-}, & \text {if } x \equiv 0(\bmod 2) \\ (x+n-2)^{-}, & \text {if } x \equiv 1(\bmod 2)\end{cases}
$$

$=$

$$
\begin{cases}\tau\left(x^{+}\right)+n, & \text { if } x \equiv 0(\bmod 2) \\ \tau\left(x^{+}\right)+n, & \text { if } x \equiv 1(\bmod 2)\end{cases}
$$


If $x=x^{-}$, then $\sigma\left(x^{-}+n\right)=\sigma\left((x+n)^{-}\right)=(x+n+1)^{-}=\sigma\left(x^{-}\right)+n$, and $\tau\left(x^{-}+n\right)=\tau\left((x+n)^{-}\right)=(x+n)^{+}=\tau\left(x^{-}\right)+n$.

Definition 2.10 Let $n$ be a positive integer. Define a relation $\tilde{n}$ on $S$ by

- $x^{+} \tilde{n} y^{+}$if $x \equiv y(\bmod 2 n)$

- $x^{-} \tilde{n} y^{-}$if $x \equiv y(\bmod 2 n)$

Theorem $2.11 \tilde{n}$ is an equivalence relation on $S$.

Proof. Trivial, since $x \equiv y(\bmod 2 n)$ is an equivalence relation on $\mathbb{Z}$.

Remark: For simplicity of notation, we write $\sim$ instead of $\tilde{n}$ when $n$ is a fixed positive integer.

Definition $2.12 S_{4 n}$ is the set of equivalence classes of $S$ under .

Example 2.13 Let [] denote an equivalence class in $S_{4 n}$. Then

- $S_{4}=\left\{\left[0^{+}\right],\left[1^{+}\right],\left[0^{-}\right],\left[1^{-}\right]\right\}$

- $S_{8}=\left\{\left[0^{+}\right],\left[1^{+}\right],\left[2^{+}\right],\left[3^{+}\right],\left[0^{-}\right],\left[1^{-}\right],\left[2^{-}\right],\left[3^{-}\right]\right\}$

Theorem 2.14 Let $x, y \in S$. If $x^{\sim} y$, then $\sigma(x)^{\sim} \sigma(y)$ and $\tau(x)^{\sim} \tau(y)$. Therefore, $\sigma, \tau$ induce permutations of $S_{4 n}$.

Proof. Suppose $x^{+^{\sim}} y^{+}$. Then $x \equiv y(\bmod 2 n)$, so $x^{+}=y^{+}+2 k n$ for some $k \in \mathbb{Z}$. By Theorem 2.9, $\sigma\left(x^{+}\right)=\sigma\left(y^{+}+2 k n\right)=\sigma\left(y^{+}\right)+2 k n$, and $\tau\left(x^{+}\right)=\tau\left(y^{+}+2 k n\right)=\tau\left(y^{+}\right)+2 k n$. Therefore, $\sigma\left(x^{+}\right)^{\sim} \sigma\left(y^{+}\right)$and $\tau\left(x^{+}\right) \tau\left(y^{+}\right)$; an identical argument applies to $x^{-\sim} y^{-}$. Therefore, $\sigma, \tau$ induce maps of $S_{4 n}$ to $S_{4 n}$. Since $\sigma, \tau: S \rightarrow S$ are onto, the induced maps on $S_{4 n}$ are onto.

RTS: the induced maps are 1-1. Suppose $\sigma\left(\left[x^{+}\right]\right)=\sigma\left(\left[y^{+}\right]\right)$. Then $\sigma\left(x^{+}\right) \sigma\left(y^{+}\right)$, implying, for some $k \in \mathbb{Z}, \sigma\left(x^{+}\right)=\sigma\left(y^{+}\right)+2 k n=\sigma\left(y^{+}+2 k n\right)$ by Theorem 2.2. Since $\sigma$ is $1-1, x^{+}=y^{+}+2 k n$, and $\left[x^{+}\right]=\left[y^{+}\right]$. Identical arguments apply to $\sigma\left(\left[x^{-}\right]\right)=\sigma\left(\left[y^{-}\right]\right), \tau\left(\left[x^{+}\right]\right)=\tau\left(\left[y^{+}\right]\right)$, and $\tau\left(\left[x^{-}\right]\right)=\tau\left(\left[y^{-}\right]\right)$.

Definition $2.15 G_{4 n}$ is the group of permutations of $S_{4 n}$ generated by the permutations of $\sigma$ and $\tau$.

Corollary 2.5 still applies, so that each word w in $G_{4 n}$ has an equivalent word representation $w=\sigma^{b}(\tau \sigma)^{a} \tau^{c} x$ with $a \geq 0$ and $x \in N\left(\omega_{1}, \omega_{2}\right)$. However, Theorem 2.3 no longer applies, so we require a modification.

Theorem 2.16 Let $\sigma, \tau: S_{4 n} \rightarrow S_{4 n}$ as defined above. Let $a, b, c$ be integers. Then $(\tau \sigma)^{a}=\sigma^{b} \tau^{c}$ if

1) $a \equiv b \equiv c \equiv 0(\bmod 2), \quad 2 b \equiv 0(\bmod 2 n), 2 c \equiv 0(\bmod 2 n)$, $a \equiv b+c(\bmod 2 n)$, or

2) $a \equiv b \equiv c \equiv 1(\bmod 2), 2(b+1) \equiv 0(\bmod 2 n), 2(c+1) \equiv 0(\bmod 2 n)$, $a \equiv b+c+1 \quad(\bmod 2 \mathrm{n})$.

Proof. First suppose $a \equiv 0(\bmod 2)$ and $(\tau \sigma)^{a}=\sigma^{b} \tau^{c}$. The formula for $(\tau \sigma)^{a}$ in Theorem 2.2 imply that $c \equiv 0(\bmod 2)$. Let $x \equiv 0(\bmod 2)$. Equating $(\tau \sigma)^{a}$ and $\sigma^{b} \tau^{c}$ on the classes $\left[x^{+}\right],\left[(x+1)^{+}\right],\left[x^{-}\right],\left[(x+1)^{-}\right]$.
1) $\left[(x-a)^{+}\right]=\left[(x-b+c)^{+}\right]$
2) $\left[(x+1+a)^{+}\right]=\left[(x+1-b-c)^{+}\right]$ 
3) $\left[(x+a)^{-}\right]=\left[(x+b+c)^{-}\right]$

4) $\left[(x+1-a)^{-}\right]=\left[(x+1+b-c)^{-}\right]$

5) Equations (1)-(4) imply, by Definition 2.10 , that $-a \equiv-b+c(\bmod 2 n)$

6) $a+1 \equiv-b-c+1(\bmod 2 n)$

7) $a \equiv b+c(\bmod 2 n)$

8) $-a+1 \equiv b-c+1(\bmod 2 n)$. Now, (5) and (7) imply that $2 c \equiv 0(\bmod 2 n)$. Additionally, (5) and (6) imply that $2 b \equiv 0(\bmod 2 n)$. Finally, (7) implies $a \equiv b+c(\bmod 2 n)$. Hence, $a \equiv 0(\bmod 2)$, and Equations (1)-(4) imply

9) $a \equiv b \equiv c \equiv 0(\bmod 2)$

10) $2 b \equiv 0(\bmod 2 n)$

11) $2 c \equiv 0(\bmod 2 n)$

12) $a \equiv b+c(\bmod 2 n)$

Conversely, suppose $9-12$ hold. Then, working $\bmod 2 n$,

$-a \equiv-(b+c) \equiv-b-c+2 c \equiv-b+c$, while

$a+1 \equiv b+c+1 \equiv b+c+1-2 b-2 c \equiv-b-c+1$, with $a \equiv b+c$, and thus,

$-a+1 \equiv-(b+c)+1 \equiv-b-c+1+2 b \equiv b-c+1$, so Equations (5)-(8) hold. This Implies (1)-(4), which implies $(\tau \sigma)^{a}=\sigma^{b} \tau^{c}$.

Now suppose $a \equiv 1(\bmod 2)$ and $(\tau \sigma)^{a}=\sigma^{b} \tau^{c}$. Again, by Theorem 2.2, we must have $c \equiv 1(\bmod 2)$ and the equations

1') $-a-2 \equiv(b+c+1)(\bmod 2 n)$

2') $a+1 \equiv(b-c)(\bmod 2 n)$

3') $a \equiv(-b+c-1)(\bmod 2 n)$

$\left.4^{\prime}\right)-a+3 \equiv(-b-c+2)(\bmod 2 n) ; \quad$ substituting $a \equiv c \equiv 1(\bmod 2)$ into (2') yields $2 \equiv(b-1)(\bmod 2)$, so

5) $a \equiv b \equiv c \equiv 1(\bmod 2) ;\left(1^{\prime}\right)$ and $\left(2^{\prime}\right)$ yields

6') $2(b+1) \equiv 0(\bmod 2 n) ;\left(1^{\prime}\right)$ and $\left(3^{\prime}\right)$ yields

7') $2(c+1) \equiv 0(\bmod 2 n) ;\left(4^{\prime}\right)$ yields

$\left.8^{\prime}\right) \quad a \equiv(b+c+1)(\bmod 2 n)$

Conversely, if $\left(5^{\prime}\right)-\left(8^{\prime}\right)$ hold, then, working $\bmod 2 n$,

- $-a-2 \equiv-b-c-1-2+2(b+1)+2(c+1) \equiv b+c+1$

- $a+1 \equiv b+c+1+1-2(c+1) \equiv b-c$

- $a \equiv b+c+1-2(b+1) \equiv-b+c-1$

- $-a+3 \equiv-b-c-1+3 \equiv-b-c+2$

Hence, (1')-(4') hold, which implies $(\tau \sigma)^{a}=\sigma^{b} \tau^{c}$.

Corollary $2.17 \sigma^{b}(\tau \sigma)^{a} \tau^{c}$ is the identity in $G_{4 n}$ if and only if

1) $a \equiv b \equiv c \equiv 0(\bmod 2), 2 b \equiv 0(\bmod 2 n), 2 c \equiv 0(\bmod 2 n)$, $a+b+c \equiv 0(\bmod 2 n)$, or

2) $a \equiv b \equiv c \equiv 1(\bmod 2), 2(b-1) \equiv 0(\bmod 2 n), 2(c-1) \equiv 0(\bmod 2 n)$, $a+b+c \equiv 1(\bmod 2 n)$

Proof. $\sigma^{b}(\tau \sigma)^{a} \tau^{c}$ is the identity if and only if $(\tau \sigma)^{a}=\sigma^{-b} \tau^{-c}$. Now, replace $b, c$ with $-b,-c$ in Theorem 2.14.

Corollary 2.18 If a word $\omega$ represents the identity in $G_{4 n}$, i.e., $\omega \in R\left(G_{4 n}\right)$, then $\omega=\sigma^{b}(\tau \sigma)^{a} \tau^{c} x$ where $x \in N\left(\omega_{1}, \omega_{2}\right)$ and a,b,c satisfy 
(1) or (2) of Corollary 2.17.

Proof. By Corollary 2.5, there is a word $y \in N\left(\omega_{1}, \omega_{2}\right)$ such that $\omega y=\sigma^{b}(\tau \sigma)^{a} \tau^{c}$. Hence, $\omega=\sigma^{b}(\tau \sigma)^{a} \tau^{c} y^{-1}$. Since $\omega$ and $y^{-1}$ represent the identity in $G_{4 n}, \sigma^{b}(\tau \sigma)^{a} \tau^{c}$ represents the identity, and Cor. 2.17 applies.

We can improve Corollary 2.18 as follows:

Theorem $2.19 A$ word $\omega \in R\left(G_{4 n}\right)$ if $\omega=g \sigma^{b}(\tau \sigma)^{a} \tau^{c} g^{-1} \omega^{\prime}$, where $\omega^{\prime} \in N\left(\omega_{1}, \omega_{2}\right), g=\sigma$ or 1 (i.e., empty word), and $a, b, c$ satisfy (henceforth $(*)): a \equiv b \equiv c \equiv 0(\bmod 2), 2 b \equiv 0(\bmod 2 n), 2 c \equiv 0(\bmod 2 n)$, $a+b+c \equiv 0(\bmod 2 n)$.

Proof. Suppose $\omega \in R\left(G_{4 n}\right)$. By Corollary 2.18, $\omega=\sigma^{b}(\tau \sigma)^{a} \tau^{c} x$ where $x \in N\left(\omega_{1}, \omega_{2}\right)$, and a, b, c satisfy (1) or (2) of Corollary 2.17. Suppose they satisfy (2). Making use of $\tau^{2} \sigma=\sigma \tau^{-2}$, which follows from the relator $\omega_{6}=\tau^{2} \sigma \tau^{2} \sigma^{-1}$, and $\tau^{-2} \sigma=\sigma \tau^{2}$, which follows from the relator $\omega_{8}=\tau^{-2} \sigma \tau^{-2} \sigma^{-1}$, we can move even powers of $\tau$ to the right to obtain

$$
\begin{aligned}
\omega & =\sigma^{b}(\tau \sigma)^{a} \tau^{c} x=\sigma \sigma^{b-1}(\tau \sigma)^{a} \tau \tau^{c-1} \sigma \sigma^{-1} x \\
& =\sigma \sigma^{b-1}(\tau \sigma)^{a} \tau \sigma \tau^{1-c} \sigma^{-1} x y=\sigma \sigma^{b^{\prime}}(\tau \sigma)^{a^{\prime}} \tau^{c^{\prime}} \sigma^{-1} \omega^{\prime}
\end{aligned}
$$

where $y \in N\left(\omega_{1}, \omega_{2}\right), \quad a^{\prime}=a+1, \quad b^{\prime}=b-1, \quad c^{\prime}=1-c, \quad \omega^{\prime}=x y \in N\left(\omega_{1}, \omega_{2}\right)$. Then

$$
\begin{gathered}
a^{\prime} \equiv b^{\prime} \equiv c^{\prime} \equiv 0(\bmod 2), \quad 2 b^{\prime} \equiv 0(\bmod 2 n), 2 c^{\prime} \equiv 0(\bmod 2 n), \text { and } \\
a^{\prime}+b^{\prime}+c^{\prime}=a+1+b-1+1-c=a+b+c-2(c-1)-1 \equiv 0(\bmod 2 n) .
\end{gathered}
$$

If (1) holds, we can take $g=1$ and $\omega^{\prime}=x$.

Next we determine the relators $R\left(G_{4 n}\right)$ and minimal presentations for $G_{4 n}$. We first consider $n=1$, then $n$ odd and greater than 1 , then $n$ even.

Theorem 2.20 $R\left(G_{4}\right)=N\left(\sigma^{2},(\tau \sigma)^{2}, \tau^{2}\right)$ and $\left\{\sigma^{2},(\tau \sigma)^{2}, \tau^{2}\right\}$ is a minimal presentation for $G_{4}$.

Proof. The diagram for $S_{4}$ (see Figure 2) shows that $\sigma^{2},(\tau \sigma)^{2}$, and $\tau^{2}$ all belong to $R\left(G_{4}\right)$. Therefore, $N\left(\sigma^{2},(\tau \sigma)^{2}, \tau^{2}\right) \subset R\left(G_{4}\right)$. Conversely, suppose $\omega \in R\left(G_{4}\right)$. By Theorem 2.19, $\omega=g \sigma^{b}(\tau \sigma)^{a} \tau^{c} g^{-1} \omega^{\prime}$ with $a, b, c$ even and $\omega^{\prime} \in N\left(\omega_{1}, \omega_{2}\right)$, and $g=\sigma$ or 1 . But $\omega_{1}=\tau^{-1} \sigma^{2} \tau \sigma^{2}$ and $\omega_{2}=\sigma^{-1} \tau^{2} \sigma \tau^{2}$ belong to $N\left(\sigma^{2},(\tau \sigma)^{2}, \tau^{2}\right)$, so $R\left(G_{4}\right) \subset N\left(\sigma^{2},(\tau \sigma)^{2}, \tau^{2}\right)$.

Now we show that $\left\{\sigma^{2},(\tau \sigma)^{2}, \tau^{2}\right\}$ is a minimal presentation for $G_{4}$. First, we show that $(\tau \sigma)^{2}$ cannot be expressed as a product of conjugates of $\sigma^{2}, \sigma^{-2}, \tau^{2}, \tau^{-2}$. Suppose it could, so (a) $(\tau \sigma)^{2}=c_{1} c_{2} \cdots c_{k}$, where each $c_{i}$ is a conjugate of $\sigma^{2}, \sigma^{-2}, \tau^{2}, \tau^{-2}$. Now (a) is an equation valid in the free group on the symbols $\sigma, \tau$. It must hold if $\sigma, \tau$ take values in any group $G$. Let $G$ be the permutation group on $\{1,2,3\}$ and $\sigma=(12), \tau=(23)$. Then $\sigma^{2}=\tau^{2}=i d$,

$$
\begin{aligned}
& S_{4} \\
& {\left[0^{+}\right] \rightleftharpoons\left[1^{+}\right]} \\
& \Uparrow \Downarrow \\
& {\left[0^{-}\right] \rightleftharpoons\left[1^{-}\right]}
\end{aligned}
$$

Figure 2. The diagram shows $\sigma, \tau$ on $S_{4}$. 
and $\tau \sigma=(132),(\tau \sigma)^{2}=(123)$ as permutations in $G$. In $G$, (a) becomes $(123)=i d$, which is a contradiction. Therefore, $(\tau \sigma)^{2}$ cannot have (a) as a representation in the free group of $\sigma, \tau$.

For the other two cases, take (b) $\sigma^{2}=c_{1} c_{2} \cdots c_{k}$, with $c_{i}$ conjugate in $\tau^{2},(\tau \sigma)^{2}$. Choose $\sigma=(132), \tau=(23),(\tau \sigma)=(12)$. Then (b) yields $(123)=i d$, a contradiction. Finally, take (c) $\tau^{2}=c_{1} c_{2} \cdots c_{k}, c_{i}$ conjugate in $\sigma^{2},(\tau \sigma)^{2}$. Choose $\sigma=(12), \tau=(132),(\tau \sigma)=(23)$. Then (c) yields $(123)=i d$, a contradiction. Therefore, $\left\{\sigma^{2},(\tau \sigma)^{2}, \tau^{2}\right\}$ is a minimal presentation for $G_{4}$.

Theorem 2.21 If $n$ is odd and $n>1$, then

$R\left(G_{4 n}\right)=N\left(\sigma^{2 n},(\tau \sigma)^{2 n}, \tau^{2 n}, \omega_{1}, \omega_{2}\right)$ and $\left\{\sigma^{2 n},(\tau \sigma)^{2 n}, \tau^{2 n}, \omega_{1}, \omega_{2}\right\}$ is a minimal presentation.

Proof. Clearly, $\sigma^{2 n},(\tau \sigma)^{2 n}, \tau^{2 n}$ all satisfy the hypothesis of Theorem 2.19, so they clearly belong to $R\left(G_{4 n}\right)$. Since $\omega_{1}, \omega_{2}$ also belong to $R\left(G_{4 n}\right)$, we have $N\left(\sigma^{2 n},(\tau \sigma)^{2 n}, \tau^{2 n}, \omega_{1}, \omega_{2}\right) \subset R\left(G_{4 n}\right)$.

Conversely, suppose $\omega \in R\left(G_{4 n}\right)$. By Theorem 2.19, $\omega=g \sigma^{b}(\tau \sigma)^{a} \tau^{c} g^{-1} \omega^{\prime}$, with $a, b, c$ satisfying $\left({ }^{*}\right)$, and $\omega^{\prime} \in N\left(\omega_{1}, \omega_{2}\right)$ with $g=\sigma$ or 1 . Then $a, b, c$ are multiples of $2 n$, so $\omega \in N\left(\sigma^{2 n},(\tau \sigma)^{2 n}, \tau^{2 n}, \omega_{1}, \omega_{2}\right)$.

Let $F(\sigma, \tau)$ be a free group on the symbols $\sigma, \tau$. To show $\left\{\sigma^{2 n},(\tau \sigma)^{2 n}, \tau^{2 n}, \omega_{1}, \omega_{2}\right\}$ is a minimal presentation, we argue as follows:

Let $\omega=\sigma^{i_{1}} \tau^{j_{1}} \cdots \sigma^{i_{k}} \tau^{j_{k}}$. Define $e_{\sigma}(\omega)=i_{1}+i_{2}+\cdots+i_{k}$,

$e_{\tau}(\omega)=j_{1}+j_{2}+\cdots+j_{k}$ Then $e_{\sigma}, e_{\tau}$ are well-defined for $\omega \in F(\sigma, \tau)$. Since $\omega_{1}=\tau^{-1} \sigma^{2} \tau \sigma^{2}, \omega_{2}=\sigma^{-1} \tau^{2} \sigma \tau^{2}$, we have $e_{\sigma}\left(\omega_{1}\right)=4, e_{\sigma}\left(\omega_{2}\right)=0$. Also, $e_{\sigma}\left(\sigma^{2 n}\right)=e_{\sigma}\left((\tau \sigma)^{2 n}\right)=2 n$, and $e_{\sigma}\left(\tau^{2 n}\right)=0$. If $\omega_{1} \in N\left(\sigma^{2 n},(\tau \sigma)^{2 n}, \tau^{2 n}, \omega_{2}\right)$, applying $e_{\sigma}$ to the representation for $\omega_{1}$ yields $4 \equiv 0(\bmod 2 n)$. Since $n \geq 3$, this is a contradiction. Therefore, $\omega_{1} \notin N\left(\sigma^{2 n},(\tau \sigma)^{2 n}, \tau^{2 n}, \omega_{2}\right)$. Using $e_{\tau}$ and employing a similar argument, we obtain $\omega_{2} \notin N\left(\sigma^{2 n},(\tau \sigma)^{2 n}, \tau^{2 n}, \omega_{1}\right)$.

Now suppose $(\tau \sigma)^{2 n} \in N\left(\sigma^{2 n}, \tau^{2 n}, \omega_{1}, \omega_{2}\right)$ so that $(\tau \sigma)^{2 n}=c_{1} c_{2} \cdots c_{k}$, where each $c_{i}$ is a conjugate in $N\left(\sigma^{2 n}, \tau^{2 n}, \omega_{1}, \omega_{2}\right)$. Since this equation holds in $F(\sigma, \tau)$, it most hold in any group $G$ in which $\sigma, \tau$ are assigned values. Let $G$ be the permutation group on $\{1,2, \cdots, 2 n+1\}$ and set $\sigma=(12)(34) \cdots(2 n-1,2 n)$, $\tau=(23)(45) \cdots(2 n, 2 n+1)$. Then $\sigma^{2}=\tau^{2}=i d$, so $c_{1} c_{2} \cdots c_{k}=i d$ in $G$, but $\tau \sigma=(1,3,5, \cdots,(2 n-1),(2 n+1), 2 n,(2 n-2), \cdots, 2)$, which has order $2 n+1$, so that $(\tau \sigma)^{2 n} \neq i d$ in $G$. Therefore, $(\tau \sigma)^{2 n} \notin N\left(\sigma^{2 n}, \tau^{2 n}, \omega_{1}, \omega_{2}\right)$.

To show that $\sigma^{2 n} \notin N\left(\tau^{2 n},(\tau \sigma)^{2 n}, \omega_{1}, \omega_{2}\right)$, let $G$ be as above, with $\sigma=(1,2,3, \cdots, 2 n, 2 n+1) \quad, \quad \tau=(1,2)(3,2 n+1)(4,2 n)(5,2 n-1) \cdots(n+1, n+3)$. Then $\tau \sigma=(2,2 n+1)(3,2 n) \cdots(n+1, n+2)$, while $\tau \sigma^{2}=(1,2 n+1)(2,2 n)(3,2 n-1) \cdots(n, n+2)$. Thus, $\sigma^{2 n} \neq i d$, but $\tau^{2 n}=(\tau \sigma)^{2 n}=i d, \omega_{2}=\sigma^{-1} \tau^{2} \sigma \tau^{2}=i d, \omega_{1}=\tau^{-1} \sigma^{2} \tau \sigma^{2}=i d$. Therefore, $\sigma^{2 n} \notin N\left(\tau^{2 n},(\tau \sigma)^{2 n}, \omega_{1}, \omega_{2}\right)$.

Finally, we show that $\tau^{2 n} \notin N\left(\sigma^{2 n}, \tau^{2 n}, \omega_{1}, \omega_{2}\right)$. Let $G$ be as above, with $\tau=(1,2,3, \cdots, 2 n, 2 n+1), \quad \sigma=(1,2)(3,2 n+1)(4,2 n)(5,2 n-1) \cdots(n+1, n+3)$.

Then $\tau \sigma=(1,3)(4,2 n+1)(5,2 n) \cdots(n+2, n+3)$, $\sigma \tau^{2}=(1,2 n+1)(2,2 n)(3,2 n-1) \cdots(n, n+2)$. Thus, $\sigma^{2 n}=(\tau \sigma)^{2 n}=i d$, 
$\omega_{1}=\tau^{-1} \sigma^{2} \tau \sigma^{2}=i d, \omega_{2}=\sigma^{-1} \tau^{2} \sigma \tau^{2}=i d$, but $\tau^{2 n} \neq i d$. Therefore, $\tau^{2 n} \notin N\left(\sigma^{2 n},(\tau \sigma)^{2 n}, \omega_{1}, \omega_{2}\right)$. This proves that $\left\{\sigma^{2 n},(\tau \sigma)^{2 n}, \tau^{2 n}, \omega_{1}, \omega_{2}\right\}$ is a minimal presentation for $R\left(G_{4 n}\right)$, the relators in $G_{4 n}$.

Theorem 2.22 If $n$ is even, then

$R\left(G_{4 n}\right)=N\left((\tau \sigma)^{2 n}, \sigma^{n}(\tau \sigma)^{n},(\tau \sigma)^{n} \tau^{n}, \omega_{1}, \omega_{2}\right)$.

Proof. First note that $\tau^{2 n}, \sigma^{2 n} \in N\left((\tau \sigma)^{2 n}, \sigma^{n}(\tau \sigma)^{n},(\tau \sigma)^{n} \tau^{n}, \omega_{1}, \omega_{2}\right)$, since

$\tau^{2 n}=(\tau \sigma)^{-2 n}\left[(\tau \sigma)^{n}\left[(\tau \sigma)^{n} \tau^{n}\right](\tau \sigma)^{-n}\right](\tau \sigma)^{n} \tau^{n}$ and

$\sigma^{2 n}=\sigma^{n}(\tau \sigma)^{n}\left[(\tau \sigma)^{-n}\left[\sigma^{n}(\tau \sigma)^{n}\right](\tau \sigma)^{n}\right](\tau \sigma)^{-2 n}$. Therefore it is sufficient to show that $R\left(G_{4 n}\right)=\tilde{N}=N\left(\sigma^{2 n}, \tau^{2 n},(\tau \sigma)^{2 n}, \sigma^{n}(\tau \sigma)^{n},(\tau \sigma)^{n} \tau^{n}, \omega_{1}, \omega_{2}\right)$. By

Theorem 2.19, $\omega \in R\left(G_{4 n}\right)$ if $\omega=g \sigma^{b}(\tau \sigma)^{a} \tau^{c} g^{-1} \omega^{\prime}$, with $\omega^{\prime} \in N\left(\omega_{1}, \omega_{2}\right)$, $g=\sigma \quad$ or 1 , and $a \equiv b \equiv c \equiv 0(\bmod 2), 2 b \equiv 2 c \equiv 0(\bmod 2 n)$,

$a+b+c \equiv 0(\bmod 2 n)$. The conditions on $a, b, c$ are equivalent to

$a \equiv b \equiv c \equiv 0(\bmod 2), a \equiv b \equiv c \equiv 0(\bmod n), a+b+c \equiv 0(\bmod 2 n)$. Each of the elements $\sigma^{2 n}, \tau^{2 n},(\tau \sigma)^{2 n}, \sigma^{n}(\tau \sigma)^{n},(\tau \sigma)^{n} \tau^{n}, \omega_{1}, \omega_{2}$ has the required form $\omega$ of Theorem 2.19. Therefore, $\tilde{N} \subset R\left(G_{4 n}\right)$.

Conversely, suppose $\omega \in R\left(G_{4 n}\right)$ with $\omega=g \sigma^{b}(\tau \sigma)^{a} \tau^{c} g^{-1} \omega^{\prime}$ as above. It remains to show that $\sigma^{b}(\tau \sigma)^{a} \tau^{c} \in \tilde{N}$, which would imply that $\omega \in \tilde{N}$. There are four cases.

1) $b \equiv 0(\bmod 2 n)$ and $c \equiv 0(\bmod 2 n)$

Then $a \equiv 0(\bmod 2 n)$, so $a=2 n a^{\prime}, b=2 n b^{\prime}, c=2 n c^{\prime}$, and $\sigma^{b}(\tau \sigma)^{a} \tau^{c}=\left(\sigma^{2 n}\right)^{b^{\prime}}\left[(\tau \sigma)^{2 n}\right]^{a^{\prime}}\left(\tau^{2 n}\right)^{c^{\prime}} \in \tilde{N}$.

2) $b \equiv 0(\bmod 2 n)$ and $c \equiv 0(\bmod 2 n)$

Since $a+b+c \equiv 0(\bmod 2 n)$, we must have $a \neq 0(\bmod 2 n)$. Then $a=2 n a^{\prime}+n, b=2 n b^{\prime}, c=2 n c^{\prime}+n$, so $\sigma^{b}(\tau \sigma)^{a} \tau^{c}=\left(\sigma^{2 n}\right)^{b^{\prime}}\left[(\tau \sigma)^{2 n}\right]^{a^{\prime}}\left[(\tau \sigma)^{n} \tau^{n}\right]\left(\tau^{2 n}\right)^{c^{\prime}} \in \tilde{N}$.

3) $c \equiv 0(\bmod 2 n)$ and $b \neq 0(\bmod 2 n)$

Then $a \neq 0(\bmod 2 n)$, so $a=2 n a^{\prime}+n, b=2 n b^{\prime}+n, c=2 n c^{\prime}$, and $\sigma^{b}(\tau \sigma)^{a} \tau^{c}=\left(\sigma^{2 n}\right)^{b^{\prime}}\left[\sigma^{n}(\tau \sigma)^{n}\right]\left[(\tau \sigma)^{2 n}\right]^{a^{\prime}}\left(\tau^{2 n}\right)^{c^{\prime}} \in \tilde{N}$.

4) $b \not \equiv 0(\bmod 2 n)$ and $c \neq \equiv(\bmod 2 n)$

Then $a \equiv 0(\bmod 2 n)$, so $a=2 n a^{\prime}, b=2 n b^{\prime}+n, c=2 n c^{\prime}+n$, and $\sigma^{b}(\tau \sigma)^{a} \tau^{c}=\left(\sigma^{2 n}\right)^{b^{\prime}}\left[\sigma^{n}(\tau \sigma)^{n}\right]\left[(\tau \sigma)^{2 n}\right]^{a^{\prime}-1}\left[(\tau \sigma)^{n} \tau^{n}\right]\left(\tau^{2 n}\right)^{c^{\prime}} \in \tilde{N}$.

Therefore, $R\left(G_{4 n}\right) \subset \tilde{N}$.

We can improve Theorem 2.22 with the following result.

Theorem 2.23 Let $n$ be an even positive integer. Let $G$ be any group containing elements $x, y$ such that $x^{n}(y x)^{n}=(y x)^{n} y^{n}=y^{-1} x^{2} y x^{2}=1$. Then $(y x)^{2 n}=1$.

Proof. First note that $y^{-1} x^{m} y x^{m}=1$ for all even $m>0$. This is true for $m=2$ by hypothesis, and the general result follows inductively from $y^{-1} x^{m+2} y x^{m+2}=y^{-1} x^{m} y\left(y^{-1} x^{2} y x^{2}\right) x^{m}=y^{-1} x^{m} y x^{m}=1$. Also, $x^{n}(y x)^{n}=1$ 
implies $x^{n}=(y x)^{-n}$, and $(y x)^{n} y^{n}=1$ implies $y^{n}=(y x)^{-n}$, so $x^{n}=y^{n}$ and $(y x)^{2 n}=y^{-2 n}$. Therefore, it suffices to show that $y^{2 n}=1$. But $x^{n}=y^{n}$ and $y^{-1} x^{n} y x^{n}=1$ imply that $y^{2 n}=y^{-1} y^{n} y y^{n}=y^{-1} x^{n} y x^{n}=1$.

Corollary 2.24 If $n$ is even, then $R\left(G_{4 n}\right)=N\left(\sigma^{n}(\tau \sigma)^{n},(\tau \sigma)^{n} \tau^{n}, \omega_{1}, \omega_{2}\right)$.

Proof. Since $\omega_{1}=\tau^{-1} \sigma^{2} \tau \sigma^{2}$, Theorem 2.23 implies that $(\tau \sigma)^{2 n} \in N\left(\sigma^{n}(\tau \sigma)^{n},(\tau \sigma)^{n} \tau^{n}, \omega_{1}, \omega_{2}\right)$. Therefore,

$R\left(G_{4 n}\right)=N\left(\sigma^{n}(\tau \sigma)^{n},(\tau \sigma)^{n} \tau^{n}, \omega_{1}, \omega_{2}\right)$. $\mathbf{n}$ Now we determine minimal presentation for $R\left(G_{4 n}\right)$ when $n$ is even. We start with $n=2$.

Theorem $2.25\left\{\sigma^{2}(\tau \sigma)^{2},(\tau \sigma)^{2} \tau^{2}, \omega_{1}\right\}$ is a minimal presentation for $G_{8}$.

Proof.

1) Since $\sigma^{2}=\tau^{2}$ follows from $\sigma^{2}(\tau \sigma)^{2}=(\tau \sigma)^{2} \tau^{2}=1$, this implies that $\omega_{2}=\sigma^{-1} \tau^{2} \sigma \tau^{2} \in N\left(\sigma^{2}(\tau \sigma)^{2},(\tau \sigma)^{2} \tau^{2}, \omega_{1}\right)$.

2) We have $\sigma^{2}(\tau \sigma)^{2} \notin N\left((\tau \sigma)^{2} \tau^{2}, \omega_{1}\right)$ since $e_{\tau}\left(\sigma^{2}(\tau \sigma)^{2}\right)=2$, whereas $e_{\tau}\left((\tau \sigma)^{2} \tau^{2}\right)=4$ and $e_{\tau}\left(\omega_{1}\right)=0$ are both divisible by 4 .

3) We have $(\tau \sigma)^{2} \tau^{2} \notin N\left(\sigma^{2}(\tau \sigma)^{2}, \omega_{1}\right)$ since $e_{\sigma}\left((\tau \sigma)^{2} \tau^{2}\right)=2$, whereas $e_{\sigma}\left(\sigma^{2}(\tau \sigma)^{2}\right)=4$ and $e_{\sigma}\left(\omega_{1}\right)=e_{\sigma}\left(\tau^{-1} \sigma^{2} \tau \sigma^{2}\right)=4$ are both divisible by 4 .

4) To show that $\omega_{1} \notin N\left(\sigma^{2}(\tau \sigma)^{2},(\tau \sigma)^{2} \tau^{2}\right)$, let $G$ be the permutation group on $\{1,2,3\}$ and set $\sigma=\tau=\{1,2,3\}$. Then $(\tau \sigma)^{2} \tau^{2}=\sigma^{2}(\tau \sigma)^{2}=\sigma^{6}=1$, but $\omega_{1}=\tau^{-1} \sigma^{2} \tau \sigma^{2}=\sigma^{4}=\sigma \neq 1$, so $\omega_{1} \notin N\left(\sigma^{2}(\tau \sigma)^{2},(\tau \sigma)^{2} \tau^{2}\right)$.

Theorem 2.26 If $n$ is even, $n>2$, then $\left\{\sigma^{n}(\tau \sigma)^{n},(\tau \sigma)^{n} \tau^{n}, \omega_{1}, \omega_{2}\right\}$ is a minimal presentation for $G_{4 n}$.

Proof. Corollary 2.24 states that it is a presentation. To demonstrate its minimality, we consider two cases:

1) $n \neq 0(\bmod 4)$ : In this case, $n=2 m$ with $m>1, m$ odd.

(a) $\sigma^{n}(\tau \sigma)^{n} \notin N\left((\tau \sigma)^{n} \tau^{n}, \omega_{1}, \omega_{2}\right)$

because $e_{\tau}\left(\sigma^{n}(\tau \sigma)^{n}\right)=n \equiv 0(\bmod 4)$, whereas

$$
e_{\tau}\left((\tau \sigma)^{n} \tau^{n}\right)=2 n \equiv 0(\bmod 4), e_{\tau}\left(\omega_{1}\right)=e_{\tau}\left(\tau^{-1} \sigma^{2} \tau \sigma^{2}\right)=0 \equiv 0(\bmod 4),
$$

and

$$
e_{\tau}\left(\omega_{2}\right)=e_{\tau}\left(\sigma^{-1} \tau^{2} \sigma \tau^{2}\right)=4 \equiv 0(\bmod 4)
$$

(b) $(\tau \sigma)^{n} \tau^{n} \notin N\left(\sigma^{n}(\tau \sigma)^{n}, \omega_{1}, \omega_{2}\right)$, because $e_{\sigma}\left((\tau \sigma)^{n} \tau^{n}\right)=n \equiv 0(\bmod 4)$, but $\sigma^{n}(\tau \sigma)^{n}, \omega_{1}, \omega_{2}$ all have $e_{\sigma}$ values divisible by 4 .

(c) $\omega_{1} \notin N\left(\sigma^{n}(\tau \sigma)^{n},(\tau \sigma)^{n} \tau^{n}, \omega_{2}\right)$ because $e_{\sigma}\left(\omega_{1}\right)=4 \neq 0(\bmod m)$, but the other elements have $e_{\sigma}$ values divisible by $m$.

(d) $\omega_{2} \notin N\left(\sigma^{n}(\tau \sigma)^{n},(\tau \sigma)^{n} \tau^{n}, \omega_{1}\right)$ because $e_{\tau}\left(\omega_{2}\right)=4 \equiv 0(\bmod m)$, but the other elements have $e_{\tau}$ values divisible by $m$.

2) $n \equiv 0(\bmod 4):$ In this case, $n=4 k$.

(a) $(\tau \sigma)^{n} \tau^{n} \notin N\left(\sigma^{n}(\tau \sigma)^{n}, \omega_{1}, \omega_{2}\right)$. Let $G$ be the permutation group on $\{1,2, \cdots, 2 n, \tilde{1}, \tilde{2}, \cdots, 2 n\}$. Let 
$\sigma=(1, \tilde{3}, 5, \tilde{7}, \cdots, \widetilde{2 n-1})(\tilde{1}, 3, \tilde{5}, 7, \cdots,(2 n-1))(2 n, \widetilde{2 n-2}, \cdots, \tilde{2})(\widetilde{2 n},(2 n-2), \cdots, 2)$, and $\tau=(1,2,3, \cdots, 2 n)(\widetilde{2 n}, \widetilde{2 n-1}, \cdots, \tilde{2}, \tilde{1})$. Then $\sigma^{n}=1, \tau^{n} \neq 1$. Also, $(\tau \sigma)^{2}=1$, so $(\tau \sigma)^{n}=1$. Therefore $\sigma^{n}(\tau \sigma)^{n}=1$, but $(\tau \sigma)^{n} \tau^{n}=\tau^{n} \neq 1$. Let

$$
x^{\prime}= \begin{cases}\tilde{y}, & \text { if } x=y \\ y, & \text { if } x=\tilde{y}\end{cases}
$$

Let $a+b$ denote addition $(\bmod 2 n)$.

If $x$ is odd, $x \stackrel{\sigma^{2}}{\longrightarrow} x+4 \stackrel{\tau}{\longrightarrow}(x+4) \pm 1 \stackrel{\sigma^{2}}{\longrightarrow} x \pm 1 \stackrel{\tau^{-1}}{\longrightarrow} x$

If $x$ is even, $x \stackrel{\sigma^{2}}{\longrightarrow} x-4 \stackrel{\tau}{\longrightarrow}(x-4) \pm 1 \stackrel{\sigma^{2}}{\longrightarrow} x \pm 1 \stackrel{\tau^{-1}}{\longrightarrow} x$

so $\omega_{1}=\tau^{-1} \sigma^{2} \tau \sigma^{2}=1$ in $G$.

For example, if $n=4$, then $G$ is the permutation group on $\{1,2,3, \cdots, 8, \tilde{1}, \tilde{2}, \cdots, \tilde{8}\}$.

$$
\begin{aligned}
& \sigma=(1, \tilde{3}, 5, \tilde{7})(\tilde{1}, 3, \tilde{5}, 7)(8, \tilde{6}, 4, \tilde{2})(\tilde{8}, 6, \tilde{4}, 2) \\
& \sigma^{2}=(1,5)(\tilde{3}, \tilde{7})(\tilde{1}, \tilde{5})(3,7)(8,4)(\tilde{6}, \tilde{2})(\tilde{8}, \tilde{4})(6,2) \\
& \tau=(1,2,3, \cdots, 8)(\tilde{8}, \tilde{7}, \cdots, \tilde{1}) \\
& \tau^{-1}=(8,7, \cdots, 1)(\tilde{1}, \tilde{2}, \cdots, \tilde{8}) \\
& \tau \sigma^{2}=(1,6,3,8,5,2,7,4)(\tilde{1}, \tilde{4}, \tilde{7}, \tilde{2}, \tilde{5}, \tilde{8}, \tilde{3}, \tilde{6}) \\
& \tau^{-1} \sigma^{2}=(1,4,7,2,5,8,3,6)(\tilde{1}, \tilde{6}, \tilde{3}, \tilde{8}, \tilde{5}, \tilde{2}, \tilde{7}, \tilde{4}) \\
& \text { so } \tau^{-1} \sigma^{2} \tau \sigma^{2}=1 \\
& \text { For } \omega_{2}=\sigma^{-1} \tau^{2} \sigma \tau^{2}, \text { we have: }
\end{aligned}
$$

If $x$ is odd, $x \stackrel{\tau^{2}}{\longrightarrow} x \pm 2 \stackrel{\sigma}{\longrightarrow}[(x \pm 2)+2]^{\prime} \stackrel{\tau^{2}}{\longrightarrow}(x+2)^{\prime} \stackrel{\sigma^{-1}}{\longrightarrow} x$

If $x$ is even, $x \stackrel{\tau^{2}}{\longrightarrow} x \pm 2 \stackrel{\sigma}{\rightarrow}[(x \pm 2)-2]^{\prime} \stackrel{\tau^{2}}{\longrightarrow}(x-2)^{\prime} \stackrel{\sigma^{-1}}{\longrightarrow} x$

Therefore, $\omega_{2}=1$ in $G$.

Since $\sigma^{n}(\tau \sigma)^{n}, \omega_{1}, \omega_{2}$ are equal to the identity in $G$, but $(\tau \sigma)^{n} \tau^{n} \neq 1$, it follows that $(\tau \sigma)^{n} \tau^{n} \notin N\left(\sigma^{n}(\tau \sigma)^{n}, \omega_{1}, \omega_{2}\right)$.

(b) $\sigma^{n}(\tau \sigma)^{n} \notin N\left((\tau \sigma)^{n} \tau^{n}, \omega_{1}, \omega_{2}\right)$.

Using $\sigma$ and $\tau$ from part (a), we note that $(\sigma \tau)^{2}=1$. Therefore, if we exchange the definitions of $\sigma$ and $\tau$, we obtain $\sigma^{n}(\tau \sigma)^{n} \neq 1$, but $(\tau \sigma)^{n} \tau^{n}=\omega_{1}=\omega_{2}=1$ in $G$, which proves (b).

(c) $\omega_{1} \notin N\left(\sigma^{n}(\tau \sigma)^{n},(\tau \sigma)^{n} \tau^{n}, \omega_{2}\right)$.

Recall that $n=4 k$. Let $G$ be the permutation group on $\{1,2, \cdots, 8 k\}$.

If $k=1$, let $\sigma=(1,2,3,4)(5,6,7,8), \tau=(1,5)(2,6)$

Then $\tau \sigma=(1,6,7,8)(2,3,4,5)$, so $\sigma^{4}=\tau^{4}=(\tau \sigma)^{4}=1$, but (using $\tau^{-1}=\tau$ ) $\omega_{1}(1)=\tau \sigma^{2} \tau \sigma^{2}(1)=\tau \sigma^{2} \tau(3)=\tau \sigma^{2}(3)=\tau(1)=5$.

If $k>1$, let $\sigma=(1,2, \cdots, 4 k)(4 k+1,4 k+2, \cdots, 8))$ and $\tau=(1,4 k+1)(2,4 k+2) \cdots(4 k, 4 k+4 k)$

Then $\sigma^{n}=\tau^{n}=\omega_{2}=1$.

Also,

$\tau \sigma=(1,4 k+2,3,4 k+4, \cdots, 4 k-1,4 k+4 k)(2,4 k+3,4,4 k+5, \cdots, 4 k, 4 k+1)$, so $(\tau \sigma)^{n}=1$

But $\omega_{1}(1)=\tau \sigma^{2} \tau \sigma^{2}(1)=\tau \sigma^{2} \tau(3)=\tau \sigma^{2}(4 k+3)=\tau(4 k+5)=5$. 
Therefore $\omega_{1} \notin N\left(\sigma^{n}(\tau \sigma)^{n},(\tau \sigma)^{n} \tau^{n}, \omega_{2}\right)$.

(d) $\omega_{2} \notin N\left(\sigma^{n}(\tau \sigma)^{n},(\tau \sigma)^{n} \tau^{n}, \omega_{1}\right)$. Let $G$ be defined as in part (c).

If we exchange $\sigma, \tau$ from part (c), we obtain $\omega_{2} \notin N\left(\sigma^{n}(\tau \sigma)^{n},(\tau \sigma)^{n} \tau^{n}, \omega_{1}\right)$.

In summary, minimal presentations for $R\left(G_{4 n}\right)$ are:
(a) $\left\{\sigma^{2}, \tau^{2},(\tau \sigma)^{2}\right\}$ for $n=1$;
(b) $\left\{\sigma^{2}(\tau \sigma)^{2},(\tau \sigma)^{2} \tau^{2}, \omega_{1}\right\}$ for $n=2$;
(c) $\left\{\sigma^{2 n}, \tau^{2 n},(\tau \sigma)^{2 n}, \omega_{1}, \omega_{2}\right\}$ for $n$ odd, $n>1$;
(d) $\left\{\sigma^{n}(\tau \sigma)^{n},(\tau \sigma)^{n} \tau^{n}, \omega_{1}, \omega_{2}\right\}$ for $n$ even, $n>2$.

Definition 2.27 The order of a group is the number of elements in the group. We require two lemmas to prove a theorem about the order of $G_{4 n}$ :

Lemma 2.28 The following identities hold in $G_{4 n}$ :

1) $(\tau \sigma)^{-1} \sigma^{2}=\sigma^{-2}(\tau \sigma)^{-1}$

2) $(\tau \sigma)^{-1} \sigma^{-2}=\sigma^{2}(\tau \sigma)^{-1}$

3) $\tau(\tau \sigma)^{a} \tau^{c}=\sigma^{2}(\tau \sigma)^{-1} \tau^{-1}(\tau \sigma)^{a-1} \tau^{c}, a, c \in \mathbb{Z}$

4) $\tau^{-1}(\tau \sigma)^{a} \tau^{c}=\sigma^{2}(\tau \sigma)^{-1} \tau(\tau \sigma)^{a-1} \tau^{c}, a, c \in \mathbb{Z}$

Proof (of Lemma 2.28):

1) $\omega_{1}=\tau^{-1} \sigma^{2} \tau \sigma^{2} \in R\left(G_{4 n}\right)$, so

$\tau^{-1} \sigma^{2}=\sigma^{-2} \tau^{-1} \rightarrow(\tau \sigma)^{-1} \sigma^{2}=\sigma^{-1} \tau^{-1} \sigma^{2}=\sigma^{-2} \sigma^{-1} \tau^{-1}=\sigma^{-2}(\tau \sigma)^{-1}$.

2) $\omega_{3}=\tau^{-1} \sigma^{-2} \tau \sigma^{-2} \in R\left(G_{4 n}\right)$, so

$\tau^{-1} \sigma^{-2}=\sigma^{2} \tau^{-1} \rightarrow(\tau \sigma)^{-1} \sigma^{-2}=\sigma^{-1} \tau^{-1} \sigma^{-2}=\sigma^{2} \sigma^{-1} \tau^{-1}=\sigma^{2}(\tau \sigma)^{-1}$.

3) $\tau(\tau \sigma)^{a} \tau^{c}=\tau^{2} \sigma(\tau \sigma)^{a-1} \tau^{c}=\sigma \tau^{-2}(\tau \sigma)^{a-1} \tau^{c}$ (since

$\left.\omega_{2}=\sigma^{-1} \tau^{2} \sigma \tau^{2} \in R\left(G_{4 n}\right)\right)$

$=\left[\sigma^{2}(\tau \sigma)^{-1} \tau\right] \tau^{-2}(\tau \sigma)^{a-1} \tau^{c}=\sigma^{2}(\tau \sigma)^{-1} \tau^{-1}(\tau \sigma)^{a-1} \tau^{c}$.

4) $\tau^{-1}(\tau \sigma)^{a} \tau^{c}=\sigma(\tau \sigma)^{a-1} \tau^{c}=\sigma^{2}(\tau \sigma)^{-1} \tau(\tau \sigma)^{a-1} \tau^{c}$.

Lemma 2.29 Every element $x \in G_{4 n}$ has representation $x=\sigma^{b}(\tau \sigma)^{a} \tau^{c}$ with $b$ even.

Proof (of Lemma 2.29): By Corollary 2.5, every $x \in G_{4 n}$ has a representation $x=\sigma^{b}(\tau \sigma)^{a} \tau^{c}$, with $a \geq 0$. If $b$ is even, the proof is complete. Let $b$ be odd and set $b=B+1, B$ even. Then

$x=\sigma^{B} \sigma(\tau \sigma)^{a} \tau^{c}=\sigma^{B} \sigma^{2}(\tau \sigma)^{-1} \tau\left[(\tau \sigma)^{a} \tau^{c}\right]=\sigma^{B} \sigma^{2}(\tau \sigma)^{-1} \sigma^{2}(\tau \sigma)^{-1} \tau^{-1}(\tau \sigma)^{a-1} \tau^{c}$

(by part (3) of Lemma 2.28) $=\sigma^{B} \sigma^{2}(\tau \sigma)^{-1} \sigma^{2}(\tau \sigma)^{-1} \sigma^{2}(\tau \sigma)^{-1} \tau(\tau \sigma)^{a-2} \tau^{c}$ (by part (4) of Lemma 2.28)

Continuing to apply parts (3) and (4) we obtain

$$
x= \begin{cases}\sigma^{B}\left[\sigma^{2}(\tau \sigma)^{-1}\right]^{a+1} \tau^{-1} \tau^{c}, & a \text { odd } \\ \sigma^{B}\left[\sigma^{2}(\tau \sigma)^{-1}\right]^{a+1} \tau \tau^{c}, & a \text { even }\end{cases}
$$

By parts (1) and (2) of Lemma 2.28, all powers of $\sigma^{2}$ can be commuted to the left of $(\tau \sigma)^{-1}$ while preserving even parity. Hence, $x=\sigma^{B^{\prime}}(\tau \sigma)^{-(a+1)} \tau^{c \pm 1}$ 
with $B^{\prime}$ even.

Theorem 2.30 The order of $G_{4 n}$ is:

1) $4 n^{3}$ if $n$ is odd

2) $n^{3}$ if $n$ is even.

Proof.

1) Suppose $n$ is odd. By Lemma 2.29, $x=\sigma^{b}(\tau \sigma)^{a} \tau^{c}$, with $b$ even. By Theorems 2.20 and 2.21, $\sigma^{2 n},(\tau \sigma)^{2 n}, \tau^{2 n}$ all belong to $R\left(G_{4 n}\right)$. Therefore we can take $x=\sigma^{\bar{b}}(\tau \sigma)^{\bar{a}} \tau^{\bar{c}}$, with $0 \leq \bar{a}, \bar{b}, \bar{c}<2 n$, and $\bar{b}$ even. There are $(2 n)(n)(2 n)=4 n^{3}$ possibilities for $\bar{a}, \bar{b}, \bar{c}$. If $\sigma^{\bar{b}_{1}}(\tau \sigma)^{\bar{a}_{1}} \tau^{\bar{c}_{1}}=\sigma^{\bar{b}_{2}}(\tau \sigma)^{\bar{a}_{2}} \tau^{\bar{c}_{2}}$, then $(\tau \sigma)^{-\bar{a}_{2}} \sigma^{\bar{b}_{1}-\bar{b}_{2}}(\tau \sigma)^{\bar{a}_{1}} \tau^{\bar{c}_{1}-\bar{c}_{2}}=1$. Since $\bar{b}_{1}-\bar{b}_{2}$ is even, we can use the commutation rules to obtain $\sigma^{ \pm\left(\bar{b}_{1}-\bar{b}_{2}\right)}(\tau \sigma)^{\bar{a}_{1}-\bar{a}_{2}} \tau^{\bar{c}_{1}-\bar{c}_{2}}=1$.

Also, Corollary 2.17 implies that $\bar{a}_{1}-\bar{a}_{2}$ and $\bar{c}_{1}-\bar{c}_{2}$ are both even, and

- $\bar{b}_{1}-\bar{b}_{2} \equiv 0(\bmod n)$

- $\bar{c}_{1}-\bar{c}_{2} \equiv 0(\bmod n)$

- $\bar{a}_{1}-\bar{a}_{2} \equiv 0(\bmod n)$.

Since $\bar{b}_{1}-\bar{b}_{2} \equiv \bar{c}_{1}-\bar{c}_{2} \equiv \bar{a}_{1}-\bar{a}_{2} \equiv 2(\bmod n)$, and $n$ is odd, this implies

- $\bar{b}_{1}-\bar{b}_{2} \equiv 0(\bmod 2 n)$

- $\bar{c}_{1}-\bar{c}_{2} \equiv 0(\bmod 2 n)$

- $\bar{a}_{1}-\bar{a}_{2} \equiv 0(\bmod 2 n)$,

which implies $\bar{b}_{1}=\bar{b}_{2}, \bar{c}_{1}=\bar{c}_{2}, \bar{a}_{1}=\bar{a}_{2}$ because of the conditions on $\bar{a}_{i}, \bar{b}_{i}, \bar{c}_{i}$. Therefore the representation $\sigma^{\bar{b}}(\tau \sigma)^{\bar{a}} \tau^{\bar{c}}$ is unique, and $G_{4 n}$ contains $4 n^{3}$ elements when $n$ is odd.

2) Now assume $n$ even. Again, let $x=\sigma^{b}(\tau \sigma)^{a} \tau^{c}$, with $b$ even.

By Theorem 2.19, $\sigma^{n}(\tau \sigma)^{n},(\tau \sigma)^{n} \tau^{n},(\tau \sigma)^{2 n}$ belong to $R\left(G_{4 n}\right)$. Using $\sigma^{n}=(\tau \sigma)^{-n}$, we can transform $x$ so that $0 \leq b<n$ and $b$ is even. Using $(\tau \sigma)^{n}=\tau^{-n}$, we can transform $x$ so that $0 \leq a<n$. Finally, using $(\tau \sigma)^{2 n}=1$, we can transform $x$ into:

$\left.{ }^{\star}\right) x=\sigma^{b}(\tau \sigma)^{a} \tau^{c}$ with $0 \leq b<n, 0 \leq a<n, 0 \leq c<2 n, b$ even.

The total number of choices for $a, b, c$ is $n\left(\frac{n}{2}\right)(2 n)=n^{3}$. The choices yield distinct elements of $G_{4 n}$, for if $\sigma^{\bar{b}_{1}}(\tau \sigma)^{\overline{a_{2}}} \tau^{\bar{c}_{1}}=\sigma^{\bar{b}_{2}}(\tau \sigma)^{\bar{a}_{2}} \tau^{\bar{c}_{2}}$, then $(\tau \sigma)^{-\bar{a}_{2}} \sigma^{\bar{b}_{1}-\bar{b}_{2}}(\tau \sigma)^{\bar{a}_{1}} \tau^{\bar{c}_{1}-\bar{c}_{2}}=1$, and by commuting even powers of $\sigma$ with $(\tau \sigma)^{ \pm 1}$, we obtain $\sigma^{ \pm\left(\bar{b}_{1}-\bar{b}_{2}\right)}(\tau \sigma)^{\bar{a}_{1}-\bar{a}_{2}} \tau^{\bar{c}_{1}-\bar{c}_{2}}=1$. By Condition (1) of Corollary 2.17, we obtain

- $\bar{b}_{1}-\bar{b}_{2} \equiv 0(\bmod n)$

- $\bar{c}_{1}-\bar{c}_{2} \equiv 0(\bmod n)$

- $\bar{a}_{1}-\bar{a}_{2} \equiv 0(\bmod n)$.

Since $0 \leq\left|\bar{a}_{1}-\bar{a}_{2}\right|,\left|\bar{b}_{1}-\bar{b}_{2}\right|<n$, we must have $\bar{b}_{1}=\bar{b}_{2}$ and $\bar{a}_{1}=\bar{a}_{2}$. However, by Condition (1) of Corollary $2.17, \bar{c}_{1}-\bar{c}_{2} \equiv 0(\bmod 2 n)$, so $\bar{c}_{1}=\bar{c}_{2}$, since $0 \leq\left|\overline{c_{1}}-\overline{c_{2}}\right|<2 n$. Therefore, all the elements $x=\sigma^{b}(\tau \sigma)^{a} \tau^{c}$ in $\left(^{*}\right)$ are distinct and the order of $G_{4 n}$ is $n^{3}$.

Finally, we determine isomorphic group structures for $G_{4}$ and $G_{8}$.

Theorem 2.31

1) $G_{4}$ is isomorphic to the Klein-4 group $\mathbb{V}$.

2) $G_{8}$ is isomorphic to the multiplicative quaternion group $\mathbb{Q}$. 


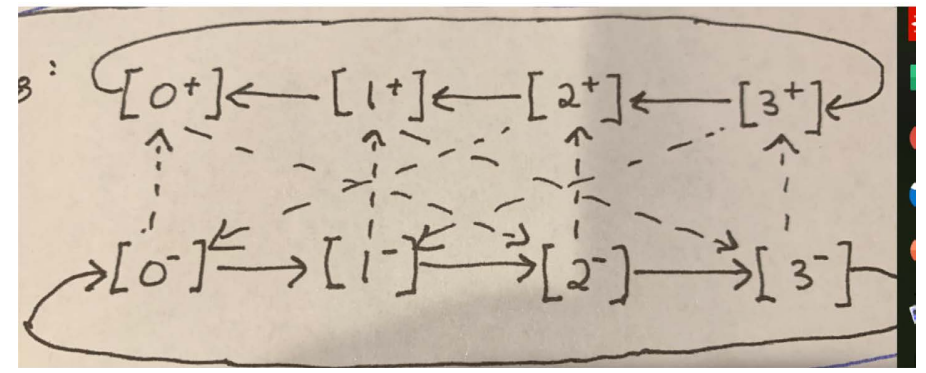

Figure 3. The diagram shows $\sigma, \tau$ on $S_{8}$.

Proof.

1) The diagram for $G_{4}$ is:

( $\sigma$ is single line, $\tau$ is double line).

$$
\begin{gathered}
{\left[0^{+}\right] \rightleftharpoons\left[1^{+}\right]} \\
\Uparrow \Downarrow \\
{\left[0^{-}\right] \rightleftharpoons\left[1^{-}\right]}
\end{gathered}
$$

By Theorem 2.30, $G_{4}$ has order 4, so $G_{4}=\{1, \sigma, \tau, \sigma \tau=\tau \sigma\}$. Since $\sigma^{2}=\tau^{2}=(\sigma \tau)^{2}=1, G_{4}$ is Abelian, but not cyclic, it is $\mathbb{V}$.

2) Quaternions $\mathbb{Q}=\{1, i, j, k,-1,-i,-j,-k\}$, with

- $\quad i j=k$

- $j i=-k$

- $i^{2}=j^{2}=k^{2}=-1$

- $j k=i$

- $k j=-i$

- $k i=j$

- $i k=-j$

each has a unique representation of the form $i^{a} j^{b}$, with $0 \leq a \leq 3,0 \leq j \leq 1$. Also, $j i=-k=i^{2} i j=i^{3} j$. The diagram for $G_{8}$ is shown in Figure 3.

Clearly, $\sigma^{2}=\tau^{2}$, and each $x \in G_{8}$ has a representation $x=\sigma^{a} \tau^{b}$, with $0 \leq a \leq 3, \quad 0 \leq b \leq 1$. Also, $\tau \sigma=\sigma^{3} \tau$. Therefore, $\phi: \mathbb{Q} \rightarrow G_{8}$ is an isomorphism defined by $\phi\left(i^{a} j^{b}\right)=\sigma^{a} \tau^{b}$ for $0 \leq a \leq 3,0 \leq b \leq 1$.

\section{Acknowledgements}

The authors acknowledge the assistance of Dr. Nathan Kahl in the preparation of this paper. This paper is in memory of our late coauthor and friend, Dr. Michael Miniere.

\section{Conflicts of Interest}

The authors declare no conflict of interest in regards to the publication of this paper.

\section{References}

[1] Miniere, M. (1997) Stevens Institute of Technology. Ph.D. Dissertation, Hoboken, 
New Jersey.

[2] Berry, K. and Tretkoff, M. (1994) The Monodromy Group of a Transcendental Function. Contemporary Mathematics, American Mathematical Society, 169, 107-122. https://doi.org/10.1090/conm/169/01653

[3] Katsura, T. (2008) Permutation Presentations of Modules over Finite Groups. Journal of Algebra, 319, 3653-3665. https://doi.org/10.1016/j.jalgebra.2008.01.023

[4] Hungerford, T.W. (1974) Algebra. Springer-Verlag, New York.

[5] Fraleigh, J.B. (1976) A First Course in Abstract Algebra. Addison-Wesley Publishing Company, Inc., Reading Mass. 